\title{
Stigma: winners and losers
}

\author{
John Bowis
}

Former UK MP and Health Minister and MEP for London, email johnbowis@aol.com

\begin{abstract}
- our Oscars went to the 2001 film A Beautiful Mind
about John Nash. It was a rare and beautifully observed drama about the experience of schizophrenia. It took us into that world and helped us understand both the experience and the reaction of others to it. Successful but a rarity. In straitened economic times such counters to stigma may not be top of the agenda. The stigma of mental illness is a prevalent global issue, perhaps more so in some countries and cultures than in others; therefore, the Royal College of Psychiatrists in the UK, national mental health associations and international organisations with mandates for mental health, such as the World Health Organization and the World Psychiatric Association, have an important role, if not duty, to keep up their programmes of anti-stigma activities.
\end{abstract}

We know that, in the UK, neuropsychiatric disorders are responsible for a third of disabilities, $15 \%$ of in-patient costs, nearly a quarter of drug costs, half the case-load of our social workers and over 90 million days lost at work a year. One in three people going to their general practitioner has mental health problems, and one in six is diagnosed with a mental disorder. That does not mean that two in three of us will have good mental health; it means we may not need, or we may not seek, or we may not be offered, treatment or care, or even advice.

Stigma may cause us to be given inappropriate treatment, with drugs and therapies and institutions and laws that at best may do no harm and at worst may lead to physical and psychological damage. It almost certainly means that we are labelled, patronised, despised, feared and, to a greater or lesser extent, segregated - in society, within our family, at work, at play and even within our health and social services.

When I first encountered mental health policy as a member of a Community Health Council, mental healthcare still featured locks, bolts, restraint garments and isolation rooms. In reality, mental health services were on the move, with more humane surroundings, treatments and therapies, and patients were on the move too - to community housing or back to their own homes. The public did not always like that. No longer 'out of sight, out of mind', it became 'out of his or her mind and living next door to me and my children'. Doctors and nurses were on the move too - with community health work, sharing patients with social workers or housing officers. Many did not much like the change either. It can be lonely for a young mental health nurse to be called out at night to a disturbed patient on an estate of tower blocks and you miss the team support of working in a hospital. Nor were all doctors happy to share knowledge of a patient with a social worker or housing officer, and vice versa.

If modern humane mental health policy is to work, it must offer a spectrum of care, from secure units to home support, and it must have the range of health and social services, housing, training, transport and benefits services working in partnership; it must have trust between professionals, patients and families and it needs government resources. All this requires political awareness, public consent and professional support. A service that does not gain professional, public and political support fails to treat and care adequately and prompts a downward spiral of public confidence, thus reinforcing stigma.

In the countries of the European Union we like to think we have moved on from the human rights abuses of patients with a mental illness. We still, though, have debates about compulsory treatment; discharge or sectioning decisions are sometimes unsound; patient abuse is from time to time exposed in a residential home; arguments abound on the vexed and conflicting rights of patients, families and communities. But, by and large, we do have fewer locks and bolts, more patient choice and consent, and legal checks and balances to see the patient's civil rights are not abused. We have not had the use of psychiatry for political purposes, as happened under the Soviet system; we do not acknowledge politically convenient diagnoses such as 'sluggish schizophrenia'; we do not pump healthy people full of drugs and turn them into zombies because of their criminal, antisocial or political behaviour.

Yet stigma is rampant in all our countries and it is a human rights abuse - unintentional, born of fear and ignorance, but just as damaging to the individual as any other form of abuse. We all contribute to the stigmatisation of people who, if they had a physical problem, would receive our sympathy and support. Yet with mental illness we so often turn away with a shiver of rejection and hope someone else will cope. Living with mental illness is tough enough, without having added to the burden of illness the pain of rejection and stigma.

To tackle it, we must confront stigma for what it is. It is prejudice. Prejudice literally means pre-judging. It uses neither logic nor facts; it is ignorance mixed with assumption and emotion. So we could start by listening to and learning from service users. They should be partners and not just patients. Then we could admit our role in stigma, in ourselves and in society. Just as we have acknowledged the concept of institutionalised racism, so we should admit to its health cousin, 'institutionalised stigma', in our political, social and health systems. We have laws on the former. We have legislated on discrimination against people with physical disabilities. Perhaps we need to explore whether such laws could apply effectively, or more effectively, to people with mental illness.

But laws will not cure prejudice: they can only try to prevent prejudiced actions. We need to educate and inform so that we can break the vicious chain of prejudice and ignorance that links public, media, patient and government. 
When I was a Health Minister in the 1990s we 'benefited' from the publicity surrounding one man jumping into the lions' den at London Zoo and another stabbing a stranger to death on the London Underground. We benefited because the shock-horror media stories led to public, Parliament and press demanding action. Indeed, each case of violence had a triple impact. The media gave dramatic coverage first to the event, then 6 months later to the trial and then another 6 months on to the inquiry report in the purple prose of Louis Blom-Cooper and others. So the public thought the number of cases was multiplying and turned against care in the community. The result was more money from finance ministers for better health and care services, but at a price the terrible stigmatising price of lost public confidence and a demand to reverse the policy of community treatment and care.

\section{Mental health suffers a quadruple whammy}

There is constant public, professional and media pressure on government and health service managers to do more, spend more, achieve more. Unlike with heart disease or AIDS or cancer, though, with mental health problems there is little political, press or public understanding of what can be done to treat, cure and rehabilitate. There is even less understanding of what can be done to prevent mental illness and promote mental health. And there are few outcome measurements that health departments and managers, much less public and politicians, can understand. There is little point in campaigning to put mental health up the political and budgeting agenda if the minister cannot convincingly argue to his colleagues that $€ x$ million invested will lead to a $y \%$ improvement in problem $z$.
If you accept that stigma was there in and around the old institutionalised regimens but that it is multiplied 100 times when you move to treatment and care in the community and attempt to integrate people with community, working and leisure life, then it becomes 100 times more important to convince people that the policy can work. If you place people neatly into a hospital ward, politicians, press and public feel you have dealt with a health problem and will not enquire too closely what you are achieving behind the hospital gates. If people are visible, then so is the nature of their health problem and questions are asked as to whether the policy is working, how much is being spent and what it is achieving.

So services need to be as visible as patients; their regimens need to be as well understood as are the illnesses; and the safety net needs to be accessible and to build confidence for patient and community alike. An effective and comprehensive spectrum of services; outcome measurements that are more easily understood by lay people; visible and responsive local services; rehabilitation and retraining services that bring people back, at a pace appropriate to each, to active and involved life, so that their friends, family and neighbours can see the progress - all will aid the defeat of stigma.

But the twin peaks are to convince the public to believe and to convince governments to spend. And they are interdependent. If the public believe, they will put pressure on the government to spend. If the government spends, it will make public belief possible.

\section{Further reading}

Ramsey, R., Page, A., Goodman, T., et al (eds) (2002) Changing Minds: Our Lives and Mental IIIness. Royal College of Psychiatrists.

The College anti-stigma campaign has compiled a good online list of sources at http://www.rcpsych.ac.uk/campaigns/changingminds/

\section{Empowering people with intellectual disability}

\section{David Skuse}

Behavioural and Brain Sciences Unit, Institute of Child Health, London, UK, email dskuse@ich.ucl.ac.uk

\begin{abstract}
ervices for people with intellectual disabilities are not necessarily a high priority even in the high-income countries. We look in this issue at the way in which approaches to support are provided in three contrasting regions, with different cultures and histories. We begin with an account of intellectual disability in India, where Dr Satish Chandra Girimaji discusses past and present frameworks for care and education. It is a cultural norm within the subcontinent that families are expected to look after relatives with disabilities, both in childhood and in adulthood; it seems that this long-standing
\end{abstract}

tradition continues. On the other hand, rapid industrialisation and urbanisation mean that it is ever more difficult for families to cope with disability, because of other pressures, without externally provided support services. The tension between tradition and economic development is palpable.

In our second paper, by Dr Meera Roy and Sivasankaran Balaratnasingam, the plight of Australian Aborigines is discussed sympathetically. Here we have a culture that is little understood it seems, even in Australia, and the closest many readers will have come to appreciating the complexity of Aboriginal culture is through books like Bruce Chatwin's Songlines. The 